\title{
Local Fractional Laplace Variational Iteration Method for Nonhomogeneous Heat Equations Arising in Fractal Heat Flow
}

\author{
Shu Xu, ${ }^{1,2}$ Xiang Ling, ${ }^{1}$ Carlo Cattani, ${ }^{3}$ Gong-Nan Xie, ${ }^{4}$ Xiao-Jun Yang, ${ }^{5}$ and Yang Zhao ${ }^{6}$ \\ ${ }^{1}$ School of Mechanical and Power Engineering, Nanjing University of Technology, Nanjing 210009, China \\ ${ }^{2}$ School of Mechanical Engineering, Huaihai Institute of Technology, Lianyungang 222005, China \\ ${ }^{3}$ Department of Mathematics, University of Salerno, Via Giovanni Paolo II, Fisciano, 84084 Salerno, Italy \\ ${ }^{4}$ School of Mechanical Engineering, Northwestern Polytechnical University, Xian, Shaanxi 710072, China \\ ${ }^{5}$ Department of Mathematics and Mechanics, China University of Mining and Technology, Xuzhou, Jiangsu 221008, China \\ ${ }^{6}$ Electronic and Information Technology Department, Jiangmen Polytechnic, Jiangmen 529090, China \\ Correspondence should be addressed to Yang Zhao; zhaoyang19781023@gmail.com
}

Received 29 May 2014; Accepted 1 July 2014; Published 7 August 2014

Academic Editor: Jun Liu

Copyright (C) 2014 Shu Xu et al. This is an open access article distributed under the Creative Commons Attribution License, which permits unrestricted use, distribution, and reproduction in any medium, provided the original work is properly cited.

\begin{abstract}
The local fractional Laplace variational iteration method is used for solving the nonhomogeneous heat equations arising in the fractal heat flow. The approximate solutions are nondifferentiable functions and their plots are also given to show the accuracy and efficiency to implement the previous method.
\end{abstract}

\section{Introduction}

Fractional calculus [1-4] was used to deal with the heat conduction equation in fractal media. Fractional heat conduction equation was studied by many researchers [5-17]. For example, Povstenko considered the thermoelasticity based on the fractional heat conduction equation [7]. Youssef suggested the generalized theory of fractional-order thermoelasticity [8]. Ezzat and El-Karamany presented the fractional-order conduction in thermoelastic medium [9]. Ezzat proposed the fractional-order heat transfer in thermoelectric fluid [10]. Sherief et al. reported the fractional-order generalized thermoelasticity with one relaxation time [11]. Vázquez et al. used the second law of thermodynamics to fractional heat conduction equation [12]. Hristov considered the inverse Stefan problem and nonlinear heat conduction with Jeffrey's fading memory by using the heat balance integral method $[13,14]$. Davey and Prosser gave the solutions of the heat transfer on fractal and prefractal domains [15]. OstojaStarzewski investigated thermoelasticity of fractal media [16]. Qi and Jiang discussed space-time fractional Cattaneo diffusion equation [17]. Bhrawy and Alghamdi applied the Legendre tau-spectral method to find time fractional heat equation with nonlocal conditions [18]. Atangana and Kılıçman suggested the Sumudu transform solving certain nonlinear fractional heat-like equations [19].

Recently, the local fractional calculus [20-22] was used to deal with the discontinuous problem for heat transfer in fractal media [23-25]. The nonhomogeneous heat equations arising in fractal heat flow were considered by using the local fractional Fourier series method [26]. The local fractional heat conduction equation was investigated by the local fractional variation iteration method [27]. The nondifferentiable solution of one-dimensional heat equations arising in fractal transient conduction was found by the local fractional Adomian decomposition method [28]. Local fractional Laplace variational iteration method $[29,30]$ was considered to deal with linear partial differential equations. In this paper, our aim is to investigate the nonhomogeneous heat equations arising in heat flow with local fractional derivative. The paper is organized as follows. Section 2 introduces the nonhomogeneous heat equations arising in heat flow with local fractional derivative. In Section 3, local fractional Laplace variational iteration method is presented. In Section 4, the nondifferentiable solutions for nonhomogeneous heat equations 
arising in heat flow with local fractional derivative are investigated. Finally, conclusions are shown in Section 5.

\section{The Nonhomogeneous Heat Equations Arising in Heat Flow with Local Fractional Derivatives}

In this section we present the one-dimensional nonhomogeneous heat equations arising in heat flow with local fractional derivatives.

Let the local fractional volume integral of the function $\mathbf{u}$ be defined as [19]

$$
\iiint \mathbf{u}\left(r_{P}\right) d \Omega^{(\gamma)}=\lim _{N \rightarrow \infty} \sum_{P=1}^{N} \mathbf{u}\left(r_{P}\right) \Delta \Omega_{P}^{(\gamma)},
$$

where the elements of the volume $\Delta \Omega_{P}^{(\gamma)} \rightarrow 0$ as $N \rightarrow$ $\infty$ and the fractal dimension of the volume $\gamma$. The equality $u(x, y, z, t)$ is the temperature at the point $(x, y, z) \in \Omega$, time $t \in T$, and the total amount of heat $H(t)$ is described as

$$
H(t)=\iiint c_{\alpha} \rho_{\alpha} u(x, y, z, t) d \Omega^{(\gamma)}
$$

where $c_{\alpha}$ is the special heat of the fractal material and $\rho_{\alpha}$ is the density of the fractal material.

The local fractional surface integral is defined as $[19,22]$

$$
\iint \mathbf{u}\left(r_{P}\right) \cdot d \mathbf{S}^{(\beta)}=\lim _{N \rightarrow \infty} \sum_{P=1}^{N} \mathbf{u}\left(r_{P}\right) \cdot \mathbf{n}_{P} \Delta S_{P}^{(\beta)},
$$

where $N$ are elements of area with a unit normal local fractional vector $\mathbf{n}_{P}, \Delta S_{P}^{(\beta)} \rightarrow 0$ as $N \rightarrow \infty$ for $\gamma=(3 / 2) \beta=$ $3 \alpha$.

From (3) the local fractional Fourier law of the material in fractal media $[19,23]$ was suggested as follows:

$$
\frac{d^{\alpha}}{d t^{\alpha}} H(t)=\oiint_{\partial \Omega^{(\beta)}} k^{2 \alpha} \nabla^{\alpha} u(x, y, z, t) \cdot d \mathbf{S}^{(\beta)},
$$

where $d \mathbf{S}^{(\beta)}$ is the fractal surface measure over $\Omega^{(\gamma)}$ and $k^{2 \alpha}$ is the thermal conductivity of the fractal material.

In view of (4), the change in heat reads as follows $[19,23]$ :

$$
\frac{d^{\alpha}}{d t^{\alpha}} H(t)=\iiint c_{\alpha} \rho_{\alpha} u_{t}^{(\alpha)}(x, y, z, t) d \Omega^{(\gamma)},
$$

where $\partial \Omega^{(\beta)}$ is the boundary of $\Omega^{(\gamma)}$.

From (2) we suggest the following source term [23]:

$$
G(t)=\iiint g(x, y, z, t) d \Omega^{(\gamma)} .
$$

Making use of (4), (5), and (6), we have

$$
\begin{aligned}
& \iiint c_{\alpha} \rho_{\alpha} u_{t}^{(\alpha)}(x, y, z, t) d \Omega^{(\gamma)} \\
& =\oiint_{\partial \Omega^{(\beta)}} k^{2 \alpha} \nabla^{\alpha} u(x, y, z, t) \cdot d \mathbf{S}^{(\beta)} \\
& \quad+\iiint g(x, y, z, t) d \Omega^{(\gamma)}
\end{aligned}
$$

such that

$$
\begin{gathered}
\iiint\left\{c_{\alpha} \rho_{\alpha} u_{t}^{(\alpha)}(x, y, z, t)-\nabla^{\alpha}\right. \\
\cdot\left[k^{2 \alpha} \nabla^{\alpha} u(x, y, z, t)\right] \\
-g(x, y, z, t)\} d \Omega^{(\gamma)}=0,
\end{gathered}
$$

which leads to the nonhomogeneous local fractional heat equations [23]:

$$
\begin{aligned}
& c_{\alpha} \rho_{\alpha} u_{t}^{(\alpha)}(x, y, z, t)-\nabla^{\alpha} \cdot\left[k^{2 \alpha} \nabla^{\alpha} u(x, y, z, t)\right] \\
& =g(x, y, z, t) .
\end{aligned}
$$

From (9) we obtain the nonhomogeneous heat equations in the dimensionless case:

$$
\phi_{t}^{(\alpha)}(x, y, z, t)-\nabla^{2 \alpha} \phi(x, y, z, t)=\varphi(x, y, z, t) .
$$

The two-dimensional case is [23]

$$
\phi_{t}^{(\alpha)}(x, y, t)-\nabla^{2 \alpha} \phi(x, y, t)=\varphi(x, y, t),
$$

and the one-dimensional case is [26]

$$
\phi_{t}^{(\alpha)}(x, t)-\phi_{x}^{(2 \alpha)}(x, t)=\varphi(x, t) .
$$

\section{Local Fractional Laplace Variational Iteration Method}

In this section, we give the idea of local fractional Laplace variational method $[29,30]$ in order to investigate the onedimensional nonhomogeneous heat equations arising in fractal heat flow.

We present the following local fractional differential operator as follows:

$$
L_{\alpha} u-R_{\alpha} u=0
$$

where the linear local fractional differential operator denotes $L_{\alpha}=d^{2 \alpha} / d x^{2 \alpha}$ and $u(x)$ is a nondifferential function.

We can write the local fractional functional formula as

$$
\begin{aligned}
u_{n+1}(x)= & u_{n}(x) \\
& +{ }_{0} I_{x}^{(\alpha)}\left\{\frac{\lambda(x-t)^{\alpha}}{\Gamma(1+\alpha)}\left[L_{\alpha} u_{n}(t)-R_{\alpha} u_{n}\right]\right\} .
\end{aligned}
$$

The local fractional Laplace transform is given as [29-32]

$$
\begin{aligned}
& \widetilde{L}_{\alpha}\{f(x)\} \\
& =f_{s}^{\widetilde{L}, \alpha}(s) \\
& =\frac{1}{\Gamma(1+\alpha)} \int_{0}^{\infty} E_{\alpha}\left(-s^{\alpha} x^{\alpha}\right) f(x)(d x)^{\alpha}, \\
& \quad 0<\alpha \leq 1,
\end{aligned}
$$


and the inverse formula of local fractional Laplace transform is suggested as [29-32]

$$
\begin{aligned}
f(x) & =\widetilde{L}_{\alpha}^{-1}\left\{f_{s}^{L, \alpha}(s)\right\} \\
& =\frac{1}{(2 \pi)^{\alpha}} \int_{\beta-i \infty}^{\beta+i \infty} E_{\alpha}\left(s^{\alpha} x^{\alpha}\right) f_{s}^{\widetilde{L}, \alpha}(s)(d s)^{\alpha},
\end{aligned}
$$

where $f(x)$ is a local fractional continuous function, $s^{\alpha}=$ $\beta^{\alpha}+i^{\alpha} \infty^{\alpha}, \operatorname{Re}\left(s^{\alpha}\right)=\beta^{\alpha}$, and the local fractional integral of $f(x)$ of order $\alpha$ in the interval $[a, b]$ is given as [23]

$$
\begin{aligned}
{ }_{a} I_{b}^{(\alpha)} f(x) & =\frac{1}{\Gamma(1+\alpha)} \int_{a}^{b} f(t)(d t)^{\alpha} \\
& =\frac{1}{\Gamma(1+\alpha)} \lim _{\Delta t \rightarrow 0} \sum_{j=0}^{j=N-1} f\left(t_{j}\right)\left(\Delta t_{j}\right)^{\alpha},
\end{aligned}
$$

with the partitions of the interval $[a, b]$ which is $\left(t_{j}, t_{j+1}\right)$, for $\Delta t_{j}=t_{j+1}-t_{j}, t_{0}=a, t_{N}=b$, and $\Delta t=\max \left\{\Delta t_{0}\right.$, $\left.\Delta t_{1}, \Delta t_{j}, \ldots\right\}, j=0, \ldots, N-1$.

From (15) the local fractional convolution of two functions is defined as [29-32]

$$
f_{1}(x) * f_{2}(x)=\frac{1}{\Gamma(1+\alpha)} \int_{-\infty}^{\infty} f_{1}(t) f_{2}(x-t)(d t)^{\alpha},
$$

and we have

$$
F_{\alpha}\left\{f_{1}(x) * f_{2}(x)\right\}=\widetilde{L}_{\alpha}\left\{f_{1}(x)\right\} \widetilde{L}_{\alpha}\left\{f_{2}(x)\right\} .
$$

From (19) we obtain

$$
\begin{aligned}
\widetilde{L}_{\alpha}\left\{u_{n+1}(x)\right\} \\
=\widetilde{L}_{\alpha}\left\{u_{n}(x)\right\} \\
\quad+\widetilde{L}_{\alpha}\left\{\frac{\lambda(x)^{\alpha}}{\Gamma(1+\alpha)}\right\} \widetilde{L}_{\alpha}\left\{L_{\alpha} u_{n}(x)-R_{\alpha} u_{n}(x)\right\} .
\end{aligned}
$$

By the local fractional variation $[23,27,29,30]$, we obtain

$$
\begin{aligned}
& \delta^{\alpha}\left\{\widetilde{L}_{\alpha}\left\{u_{n+1}(x)\right\}\right\} \\
& =\delta^{\alpha}\left\{\widetilde{L}_{\alpha}\left\{u_{n}(x)\right\}\right\} \\
& \quad+\delta^{\alpha}\left\{\widetilde{L}_{\alpha}\left\{\frac{\lambda(x)^{\alpha}}{\Gamma(1+\alpha)}\right\} \widetilde{L}_{\alpha}\left\{L_{\alpha} u_{n}(x)-R_{\alpha} u_{n}(x)\right\}\right\},
\end{aligned}
$$

which leads to

$$
\begin{aligned}
\delta^{\alpha}\left\{\widetilde{L}_{\alpha}\left\{u_{n+1}(x)\right\}\right\} \\
=\delta^{\alpha}\left\{\widetilde{L}_{\alpha}\left\{u_{n}(x)\right\}\right\} \\
+\widetilde{L}_{\alpha}\left\{\frac{\lambda(x)^{\alpha}}{\Gamma(1+\alpha)}\right\}\left\{\delta^{\alpha}\left\{\widetilde{L}_{\alpha}\left\{L_{\alpha} u_{n}(x)\right\}\right\}\right\}=0 .
\end{aligned}
$$

From (22) we have

$$
\begin{aligned}
\delta^{\alpha} & \left\{\widetilde{L}_{\alpha}\left\{L_{\alpha} u_{n}(x)\right\}\right\} \\
& =\delta^{\alpha}\left\{s^{2 \alpha} \widetilde{L}_{\alpha}\left\{u_{n}(x)\right\}-s^{\alpha} u_{n}(0)-u_{n}^{(\alpha)}(0)\right\} \\
& =s^{2 \alpha} \delta^{\alpha} \widetilde{L}_{\alpha}\left\{u_{n}(x)\right\}
\end{aligned}
$$

such that

$$
1+\widetilde{L}_{\alpha}\left\{\frac{\lambda(x)^{\alpha}}{\Gamma(1+\alpha)}\right\} s^{2 \alpha}=0 .
$$

From (24) we get

$$
\widetilde{L}_{\alpha}\left\{\frac{\lambda(x)^{\alpha}}{\Gamma(1+\alpha)}\right\}=-\frac{1}{s^{2 \alpha}}
$$

such that local fractional iteration algorithm reads as

$$
\begin{aligned}
\widetilde{L}_{\alpha}\left\{u_{n+1}(x)\right\}= & \widetilde{L}_{\alpha}\left\{u_{n}(x)\right\} \\
& -\frac{1}{s^{2 \alpha}} \widetilde{L}_{\alpha}\left\{\left(L_{\alpha} u_{n}(x)-R_{\alpha} u_{n}(x)\right)\right\},
\end{aligned}
$$

where the initial value is presented as follows:

$$
\widetilde{L}_{\alpha}\left\{u_{0}(x)\right\}=u(0)
$$

Therefore, the local fractional series solution is given as

$$
\widetilde{L}_{\alpha}\{u\}=\lim _{n \rightarrow \infty} \widetilde{L}_{\alpha}\left\{u_{n}\right\}
$$

From (28) we arrive at

$$
u=\lim _{n \rightarrow \infty} \widetilde{L}_{\alpha}^{-1}\left\{\widetilde{L}_{\alpha} u_{n}\right\}
$$

\section{The Nondifferentiable Solutions}

In this section, we discuss the one-dimensional nonhomogeneous heat equations arising in fractal heat flow.

Example 1. The nonhomogeneous local fractional heat equation with the nondifferentiable sink term is presented as follows:

$$
\begin{aligned}
& \frac{\partial^{\alpha} T(x, t)}{\partial t^{\alpha}}-\frac{\partial^{2 \alpha} T(x, t)}{\partial x^{2 \alpha}} \\
& =-\frac{x^{\alpha} E_{\alpha}\left(-t^{\alpha}\right)}{\Gamma(1+\alpha)}, \quad 0<x<1,0<t \leq 1,0<\alpha \leq 1,
\end{aligned}
$$

subject to the initial-boundary value conditions

$$
\begin{aligned}
\frac{\partial^{\alpha} T(0, t)}{\partial x^{\alpha}} & =E_{\alpha}\left(-t^{\alpha}\right), \\
T(0, t) & =0 .
\end{aligned}
$$


From (26) we obtain the local fractional iteration algorithm:

$$
\begin{aligned}
& \widetilde{L}_{\alpha}\left\{T_{n+1}(x, t)\right\}= \widetilde{L}_{\alpha}\left\{T_{n}(x, t)\right\}-\frac{1}{s^{2 \alpha}} \widetilde{L}_{\alpha} \\
& \cdot\left\{\left(\frac{\partial^{\alpha} T_{n}(x, t)}{\partial t^{\alpha}}-\frac{\partial^{2 \alpha} T_{n}(x, t)}{\partial x^{2 \alpha}}\right.\right. \\
&\left.\left.+\frac{x^{\alpha} E_{\alpha}\left(-t^{\alpha}\right)}{\Gamma(1+\alpha)}\right)\right\} \\
&= \widetilde{L}_{\alpha}\left\{T_{n}(x, t)\right\}-\frac{1}{s^{2 \alpha}} \\
& \cdot\left\{\frac{\partial^{\alpha} T_{n}(s, t)}{\partial t^{\alpha}-s^{2 \alpha} \widetilde{L}_{\alpha}\left\{T_{n}(x, t)\right\}}\right. \\
&+s^{\alpha} T_{n}(0, t)+T_{n}^{(\alpha)}(0, t) \\
&\left.+\frac{E_{\alpha}\left(-t^{\alpha}\right)}{s^{2 \alpha}}\right\} \\
&=2 \widetilde{L}_{\alpha}\left\{T_{n}(x, t)\right\}-\frac{1}{s^{2 \alpha}} \frac{\partial^{\alpha} T_{n}(s, t)}{\partial t^{\alpha}} \\
&-\frac{T_{n}^{(\alpha)}(0, t)}{s^{2 \alpha}-\frac{E_{\alpha}\left(-t^{\alpha}\right)}{s^{4 \alpha}},}
\end{aligned}
$$

where the initial value is given as

$$
\widetilde{L}_{\alpha}\left\{T_{0}(x, t)\right\}=\frac{E_{\alpha}\left(-t^{\alpha}\right)}{s^{2 \alpha}}
$$

Using (32), we have the first approximation:

$$
\begin{aligned}
\widetilde{L}_{\alpha}\left\{T_{1}(x, t)\right\}= & 2 \widetilde{L}_{\alpha}\left\{T_{0}(x, t)\right\}-\frac{1}{s^{2 \alpha}} \frac{\partial^{\alpha} T_{0}(s, t)}{\partial t^{\alpha}} \\
& -\frac{T_{0}^{(\alpha)}(0, t)}{s^{2 \alpha}}-\frac{E_{\alpha}\left(-t^{\alpha}\right)}{s^{4 \alpha}} \\
= & \frac{2 E_{\alpha}\left(-t^{\alpha}\right)}{s^{2 \alpha}}+\frac{E_{\alpha}\left(-t^{\alpha}\right)}{s^{4 \alpha}}-\frac{E_{\alpha}\left(-t^{\alpha}\right)}{s^{2 \alpha}} \\
& -\frac{E_{\alpha}\left(-t^{\alpha}\right)}{s^{4 \alpha}}=\frac{E_{\alpha}\left(-t^{\alpha}\right)}{s^{2 \alpha}} .
\end{aligned}
$$

In view of (32) and (34), we get the second approximation:

$$
\begin{aligned}
\widetilde{L}_{\alpha}\left\{T_{2}(x, t)\right\}= & 2 \widetilde{L}_{\alpha}\left\{T_{1}(x, t)\right\}-\frac{1}{s^{2 \alpha}} \frac{\partial^{\alpha} T_{1}(s, t)}{\partial t^{\alpha}} \\
& -\frac{T_{1}^{(\alpha)}(0, t)}{s^{2 \alpha}}-\frac{E_{\alpha}\left(-t^{\alpha}\right)}{s^{4 \alpha}} \\
= & \frac{2 E_{\alpha}\left(-t^{\alpha}\right)}{s^{2 \alpha}}-\frac{E_{\alpha}\left(-t^{\alpha}\right)}{s^{4 \alpha}}-\frac{E_{\alpha}\left(-t^{\alpha}\right)}{s^{2 \alpha}} \\
& -\frac{E_{\alpha}\left(-t^{\alpha}\right)}{s^{4 \alpha}}=\frac{E_{\alpha}\left(-t^{\alpha}\right)}{s^{2 \alpha}} .
\end{aligned}
$$

Making use of (32) and (35), the third approximate term reads as follows:

$$
\begin{aligned}
\widetilde{L}_{\alpha}\left\{T_{3}(x, t)\right\}= & 2 \widetilde{L}_{\alpha}\left\{T_{2}(x, t)\right\}-\frac{1}{s^{2 \alpha}} \frac{\partial^{\alpha} T_{2}(s, t)}{\partial t^{\alpha}} \\
& -\frac{T_{2}^{(\alpha)}(0, t)}{s^{2 \alpha}}-\frac{E_{\alpha}\left(-t^{\alpha}\right)}{s^{4 \alpha}} \\
= & \frac{2 E_{\alpha}\left(-t^{\alpha}\right)}{s^{2 \alpha}}-\frac{E_{\alpha}\left(-t^{\alpha}\right)}{s^{4 \alpha}}-\frac{E_{\alpha}\left(-t^{\alpha}\right)}{s^{2 \alpha}} \\
& -\frac{E_{\alpha}\left(-t^{\alpha}\right)}{s^{4 \alpha}}=\frac{E_{\alpha}\left(-t^{\alpha}\right)}{s^{2 \alpha}} .
\end{aligned}
$$

From (32) and (36), the fourth approximate term can be written as follows:

$$
\begin{aligned}
\widetilde{L}_{\alpha}\left\{T_{4}(x, t)\right\}= & 2 \widetilde{L}_{\alpha}\left\{T_{3}(x, t)\right\}-\frac{1}{s^{2 \alpha}} \frac{\partial^{\alpha} T_{3}(s, t)}{\partial t^{\alpha}} \\
& -\frac{T_{3}^{(\alpha)}(0, t)}{s^{2 \alpha}}-\frac{E_{\alpha}\left(-t^{\alpha}\right)}{s^{4 \alpha}} \\
= & \frac{2 E_{\alpha}\left(-t^{\alpha}\right)}{s^{2 \alpha}}-\frac{E_{\alpha}\left(-t^{\alpha}\right)}{s^{4 \alpha}}-\frac{E_{\alpha}\left(-t^{\alpha}\right)}{s^{2 \alpha}} \\
& -\frac{E_{\alpha}\left(-t^{\alpha}\right)}{s^{4 \alpha}}=\frac{E_{\alpha}\left(-t^{\alpha}\right)}{s^{2 \alpha}} .
\end{aligned}
$$

Making the best of (32) and (36), we can write the fifth approximate term as

$$
\begin{aligned}
\widetilde{L}_{\alpha}\left\{T_{5}(x, t)\right\}= & 2 \widetilde{L}_{\alpha}\left\{T_{4}(x, t)\right\}-\frac{1}{s^{2 \alpha}} \frac{\partial^{\alpha} T_{4}(s, t)}{\partial t^{\alpha}} \\
& -\frac{T_{4}^{(\alpha)}(0, t)}{s^{2 \alpha}}-\frac{E_{\alpha}\left(-t^{\alpha}\right)}{s^{4 \alpha}} \\
= & \frac{2 E_{\alpha}\left(-t^{\alpha}\right)}{s^{2 \alpha}}-\frac{E_{\alpha}\left(-t^{\alpha}\right)}{s^{4 \alpha}}-\frac{E_{\alpha}\left(-t^{\alpha}\right)}{s^{2 \alpha}} \\
& -\frac{E_{\alpha}\left(-t^{\alpha}\right)}{s^{4 \alpha}}=\frac{E_{\alpha}\left(-t^{\alpha}\right)}{s^{2 \alpha}} .
\end{aligned}
$$

Hence, we obtain the final term given as

$$
\widetilde{L}_{\alpha}\left\{T_{n}(x, t)\right\}=\frac{E_{\alpha}\left(-t^{\alpha}\right)}{s^{2 \alpha}} .
$$

In view of (28) and (29), we suggest the exact solution of (30) as

$$
\begin{aligned}
T(x, t) & =\lim _{n \rightarrow \infty} \widetilde{L}_{\alpha}^{-1}\left\{\widetilde{L}_{\alpha}\left\{T_{n}(x, t)\right\}\right\} \\
& =\frac{x^{\alpha}}{\Gamma(1+\alpha)} E_{\alpha}\left(-t^{\alpha}\right)
\end{aligned}
$$

and its plot is shown in Figure 1. 


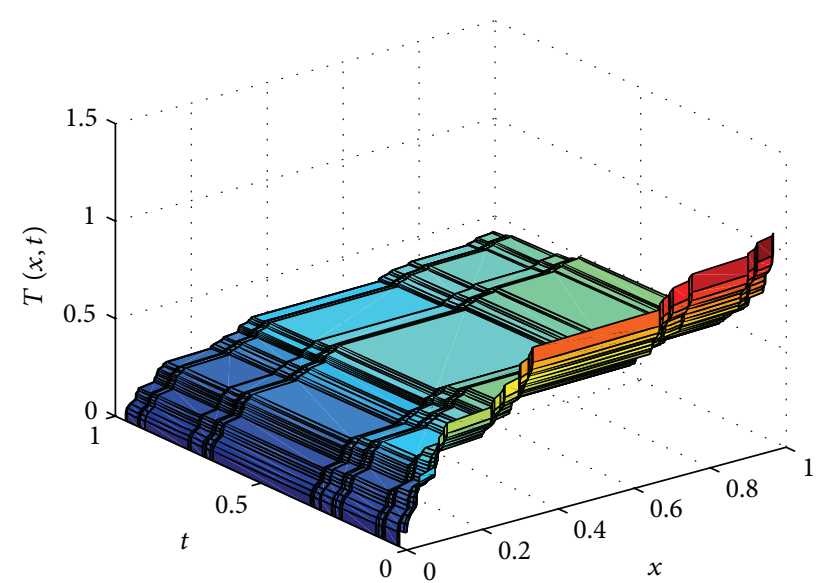

FIGURE 1: The nondifferentiable solution of nonhomogeneous local fractional heat equation with nondifferentiable sink term for $\alpha=$ $\ln 2 / \ln 3$.

Example 2. We now consider the nonhomogeneous local fractional heat equation with the nondifferentiable source term:

$$
\begin{aligned}
& \frac{\partial^{\alpha} T(x, t)}{\partial t^{\alpha}}-\frac{\partial^{2 \alpha} T(x, t)}{\partial x^{2 \alpha}} \\
& \quad=\frac{x^{\alpha} \cos _{\alpha}\left(t^{\alpha}\right)}{\Gamma(1+\alpha)}, \quad 0<x<1,0<t \leq 1,0<\alpha \leq 1,
\end{aligned}
$$

subject to the initial-boundary value conditions

$$
\begin{gathered}
\frac{\partial^{2 \alpha} T(x, t)}{\partial x^{2 \alpha}}=\sin _{\alpha}\left(t^{\alpha}\right), \\
T(0, t)=0 .
\end{gathered}
$$

In view of (26), the local fractional iteration algorithm can be structured as follows:

$$
\begin{aligned}
& \widetilde{L}_{\alpha}\left\{T_{n+1}(x, t)\right\}=\widetilde{L}_{\alpha}\left\{T_{n}(x, t)\right\}-\frac{1}{s^{2 \alpha}} \widetilde{L}_{\alpha} \\
& \left\{\left(\frac{\partial^{\alpha} T_{n}(x, t)}{\partial t^{\alpha}}-\frac{\partial^{2 \alpha} T_{n}(x, t)}{\partial x^{2 \alpha}}\right.\right. \\
& \left.\left.-\frac{x^{\alpha} \cos _{\alpha}\left(t^{\alpha}\right)}{\Gamma(1+\alpha)}\right)\right\} \\
& =\widetilde{L}_{\alpha}\left\{T_{n}(x, t)\right\}-\frac{1}{s^{2 \alpha}} \\
& \left\{\frac{\partial^{\alpha} T_{n}(s, t)}{\partial t^{\alpha}}-s^{2 \alpha} \widetilde{L}_{\alpha}\left\{T_{n}(x, t)\right\}\right. \\
& +s^{\alpha} T_{n}(0, t)+T_{n}^{(\alpha)}(0, t) \\
& \left.-\frac{\cos _{\alpha}\left(t^{\alpha}\right)}{s^{2 \alpha}}\right\}
\end{aligned}
$$

$$
\begin{aligned}
= & 2 \widetilde{L}_{\alpha}\left\{T_{n}(x, t)\right\}-\frac{1}{s^{2 \alpha}} \frac{\partial^{\alpha} T_{n}(s, t)}{\partial t^{\alpha}} \\
& -\frac{T_{n}^{(\alpha)}(0, t)}{s^{2 \alpha}}+\frac{\cos _{\alpha}\left(t^{\alpha}\right)}{s^{4 \alpha}} .
\end{aligned}
$$

Appling (43) gives the first approximate term:

$$
\begin{aligned}
\widetilde{L}_{\alpha}\left\{T_{1}(x, t)\right\}= & 2 \widetilde{L}_{\alpha}\left\{T_{0}(x, t)\right\}-\frac{1}{s^{2 \alpha}} \frac{\partial^{\alpha} T_{0}(s, t)}{\partial t^{\alpha}} \\
& -\frac{T_{0}^{(\alpha)}(0, t)}{s^{2 \alpha}}+\frac{\cos _{\alpha}\left(t^{\alpha}\right)}{s^{4 \alpha}} \\
= & \frac{2 \sin _{\alpha}\left(t^{\alpha}\right)}{s^{2 \alpha}}-\frac{\cos _{\alpha}\left(t^{\alpha}\right)}{s^{4 \alpha}} \\
& -\frac{\sin _{\alpha}\left(t^{\alpha}\right)}{s^{2 \alpha}}+\frac{E_{\alpha}\left(-t^{\alpha}\right)}{s^{4 \alpha}}=\frac{\sin _{\alpha}\left(t^{\alpha}\right)}{s^{2 \alpha}} .
\end{aligned}
$$

In view of (43) and (44), the second approximate term reads as

$$
\begin{aligned}
\widetilde{L}_{\alpha}\left\{T_{2}(x, t)\right\}= & 2 \widetilde{L}_{\alpha}\left\{T_{1}(x, t)\right\}-\frac{1}{s^{2 \alpha}} \frac{\partial^{\alpha} T_{1}(s, t)}{\partial t^{\alpha}} \\
& -\frac{T_{1}^{(\alpha)}(0, t)}{s^{2 \alpha}}+\frac{\cos _{\alpha}\left(t^{\alpha}\right)}{s^{4 \alpha}} \\
= & \frac{2 \sin _{\alpha}\left(t^{\alpha}\right)}{s^{2 \alpha}}-\frac{\cos _{\alpha}\left(t^{\alpha}\right)}{s^{4 \alpha}}-\frac{\sin _{\alpha}\left(t^{\alpha}\right)}{s^{2 \alpha}} \\
& +\frac{E_{\alpha}\left(-t^{\alpha}\right)}{s^{4 \alpha}}=\frac{\sin _{\alpha}\left(t^{\alpha}\right)}{s^{2 \alpha}} .
\end{aligned}
$$

Making use of (43) and (45), we arrive at the third approximate term:

$$
\begin{aligned}
\widetilde{L}_{\alpha}\left\{T_{3}(x, t)\right\}= & 2 \widetilde{L}_{\alpha}\left\{T_{2}(x, t)\right\}-\frac{1}{s^{2 \alpha}} \frac{\partial^{\alpha} T_{2}(s, t)}{\partial t^{\alpha}} \\
& -\frac{T_{2}^{(\alpha)}(0, t)}{s^{2 \alpha}}+\frac{\cos _{\alpha}\left(t^{\alpha}\right)}{s^{4 \alpha}} \\
= & \frac{2 \sin _{\alpha}\left(t^{\alpha}\right)}{s^{2 \alpha}}-\frac{\cos _{\alpha}\left(t^{\alpha}\right)}{s^{4 \alpha}} \\
& -\frac{\sin _{\alpha}\left(t^{\alpha}\right)}{s^{2 \alpha}}+\frac{E_{\alpha}\left(-t^{\alpha}\right)}{s^{4 \alpha}}=\frac{\sin _{\alpha}\left(t^{\alpha}\right)}{s^{2 \alpha}} .
\end{aligned}
$$

From (43) and (46) we give the fourth approximation:

$$
\begin{aligned}
\widetilde{L}_{\alpha}\left\{T_{4}(x, t)\right\}= & 2 \widetilde{L}_{\alpha}\left\{T_{3}(x, t)\right\}-\frac{1}{s^{2 \alpha}} \frac{\partial^{\alpha} T_{3}(s, t)}{\partial t^{\alpha}} \\
& -\frac{T_{3}^{(\alpha)}(0, t)}{s^{2 \alpha}}+\frac{\cos _{\alpha}\left(t^{\alpha}\right)}{s^{4 \alpha}} \\
= & \frac{2 \sin _{\alpha}\left(t^{\alpha}\right)}{s^{2 \alpha}}-\frac{\cos _{\alpha}\left(t^{\alpha}\right)}{s^{4 \alpha}}-\frac{\sin _{\alpha}\left(t^{\alpha}\right)}{s^{2 \alpha}} \\
& +\frac{E_{\alpha}\left(-t^{\alpha}\right)}{s^{4 \alpha}}=\frac{\sin _{\alpha}\left(t^{\alpha}\right)}{s^{2 \alpha}} .
\end{aligned}
$$




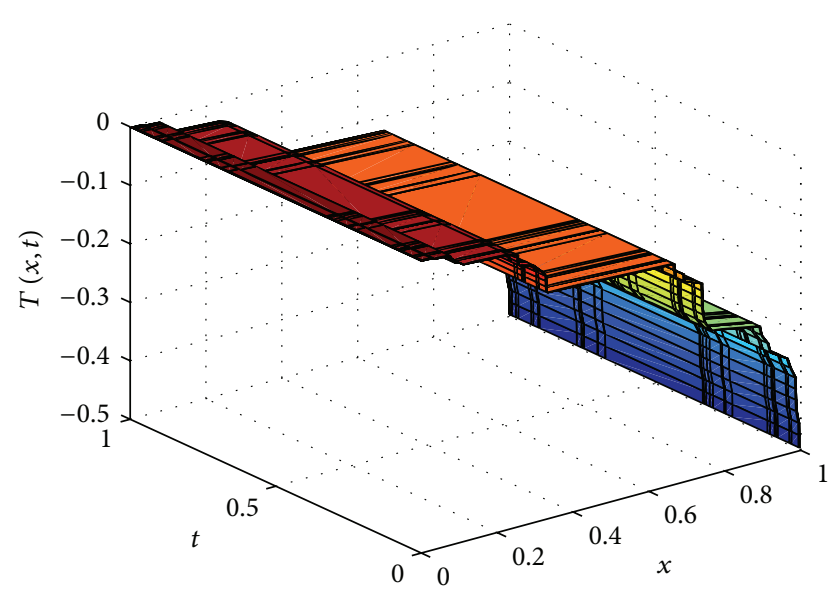

FIGURE 2: The nondifferentiable solution of the nonhomogeneous local fractional heat equation with the nondifferentiable sink term for $\alpha=\ln 2 / \ln 3$.

In view of (43) and (47), the fifth approximate term is presented as

$$
\begin{aligned}
\widetilde{L}_{\alpha}\left\{T_{5}(x, t)\right\}= & 2 \widetilde{L}_{\alpha}\left\{T_{4}(x, t)\right\}-\frac{1}{s^{2 \alpha}} \frac{\partial^{\alpha} T_{4}(s, t)}{\partial t^{\alpha}} \\
& -\frac{T_{4}^{(\alpha)}(0, t)}{s^{2 \alpha}}+\frac{\cos _{\alpha}\left(t^{\alpha}\right)}{s^{4 \alpha}} \\
= & \frac{2 \sin _{\alpha}\left(t^{\alpha}\right)}{s^{2 \alpha}}-\frac{\cos _{\alpha}\left(t^{\alpha}\right)}{s^{4 \alpha}}-\frac{\sin _{\alpha}\left(t^{\alpha}\right)}{s^{2 \alpha}} \\
& +\frac{E_{\alpha}\left(-t^{\alpha}\right)}{s^{4 \alpha}}=\frac{\sin _{\alpha}\left(t^{\alpha}\right)}{s^{2 \alpha}} .
\end{aligned}
$$

Hence, we finally have

$$
\widetilde{L}_{\alpha}\left\{T_{n}(x, t)\right\}=\frac{\sin _{\alpha}\left(t^{\alpha}\right)}{s^{2 \alpha}}
$$

so that the exact solution of nonhomogeneous local fractional heat equation with nondifferentiable source term is

$$
\begin{aligned}
T(x, t) & =\lim _{n \rightarrow \infty} \widetilde{L}_{\alpha}^{-1}\left\{\widetilde{L}_{\alpha}\left\{T_{n}(x, t)\right\}\right\} \\
& =\frac{x^{\alpha}}{\Gamma(1+\alpha)} \sin _{\alpha}\left(t^{\alpha}\right) .
\end{aligned}
$$

For the fractal dimension $\alpha=\ln 2 / \ln 3$, the plot of the nondifferentiable solution of the nonhomogeneous local fractional heat equation with the nondifferentiable source term is shown in Figure 2.

\section{Conclusions}

At the present work, the nonhomogeneous heat equations arising in the fractal heat flow were investigated. The local fractional Laplace variational iteration method was applied to obtain the nondifferentiable solutions for the nonhomogeneous local fractional heat equations with the nondifferentiable source and sink terms. Finally, the graphs of the obtained solutions are also shown.

\section{Conflict of Interests}

The authors declare that they have no conflict of interests in this paper.

\section{Acknowledgments}

The work was supported by the Natural Science Foundation of Jiangsu Colleges and Universities (no. KK-12058) and Jiangsu Province R\&D Institute of Marine Resource (no. JSIUMR 201210).

\section{References}

[1] A. A. Kilbas, H. M. Srivastava, and J. J. Trujillo, Theory and Applications of Fractional Differential Equations, vol. 204 of North-Holland Mathematics Studies, Elsevier Science, Amsterdam, The Netherlands, 2006.

[2] B. J. West, M. Bologna, and P. Grigolini, Physics of Fractal Operators, Institute for Nonlinear Science, Springer, 2003.

[3] J. Klafter, S. C. Lim, and R. Metzler, Fractional Dynamics: Recent Advances, World Scientific, 2012.

[4] D. Baleanu, J. A. T. Machado, and A. C. Luo, Fractional Dynamics and Control, Springer, New York, NY, USA, 2012.

[5] A. B. Alkhasov, R. P. Meilanov, and M. R. Shabanova, "Heat conduction equation in fractional-order derivatives," Journal of Engineering Physics and Thermophysics, vol. 84, no. 2, pp. 332341, 2011.

[6] J. M. Angulo, M. D. Ruiz-Medina, V. V. Anh, and W. Grecksch, "Fractional diffusion and fractional heat equation," Advances in Applied Probability, vol. 32, no. 4, pp. 1077-1099, 2000.

[7] Y. Z. Povstenko, "Fractional heat conduction equation and associated thermal stress," Journal of Thermal Stresses, vol. 28, no. 1, pp. 83-102, 2004.

[8] H. M. Youssef, "Theory of fractional order generalized thermoelasticity," Journal of Heat Transfer, vol. 132, no. 6, 7 pages, 2010.

[9] M. A. Ezzat and A. S. El-Karamany, "Fractional order theory of a perfect conducting thermoelastic medium," Canadian Journal of Physics, vol. 89, no. 3, pp. 311-318, 2011.

[10] M. A. Ezzat, "State space approach to thermoelectric fluid with fractional order heat transfer," Heat and Mass Transfer/Waermeund Stoffuebertragung, vol. 48, no. 1, pp. 71-82, 2012.

[11] H. H. Sherief, A. M. A. El-Sayed, and A. M. Abd El-Latief, "Fractional order theory of thermoelasticity," International Journal of Solids and Structures, vol. 47, no. 2, pp. 269-275, 2010.

[12] L. Vázquez, J. J. Trujillo, and M. P. Velasco, "Fractional heat equation and the second law of thermodynamics," Fractional Calculus and Applied Analysis, vol. 14, no. 3, pp. 334-342, 2011.

[13] J. Hristov, "An inverse stefan problem relevant to boilover: heat balance integral solutions and analysis," Thermal Science, vol. 11, no. 2, pp. 141-160, 2007.

[14] J. Hristov, "A note on the integral approach to non-linear heat conduction with Jeffrey's fading memory," Thermal Science, vol. 17, no. 3, pp. 733-737, 2013.

[15] K. Davey and R. Prosser, "Analytical solutions for heat transfer on fractal and pre-fractal domains," Applied Mathematical Modelling, vol. 37, no. 1-2, pp. 554-569, 2013.

[16] M. Ostoja-Starzewski, "Towards thermoelasticity of fractal media," Journal of Thermal Stresses, vol. 30, no. 9-10, pp. 889896, 2007. 
[17] H. Qi and X. Jiang, "Solutions of the space-time fractional Cattaneo diffusion equation," Physica A: Statistical Mechanics and its Applications, vol. 390, no. 11, pp. 1876-1883, 2011.

[18] A. H. Bhrawy and M. A. Alghamdi, "A Legendre tau-spectral method for solving time fractional heat equation with nonlocal conditions," The Scientific World Journal, vol. 2014, Article ID 706296, 7 pages, 2014.

[19] A. Atangana and A. Kılıçman, "The use of Sumudu transform for solving certain nonlinear fractional heat-like equations," Abstract and Applied Analysis, vol. 2013, Article ID 737481, 12 pages, 2013.

[20] X. J. Yang, D. Baleanu, and J. A. T. Machado, "Mathematical aspects of the Heisenberg uncertainty principle within local fractional Fourier analysis," Boundary Value Problems, vol. 2013, article 131, 2013.

[21] X. J. Yang, D. Baleanu, and W. P. Zhong, "Approximate solutions for diffusion equations on cantor space-time," Proceedings of the Romanian Academy A, vol. 14, no. 2, pp. 127-133, 2013.

[22] X. Yang, D. Baleanu, and J. A. Tenreiro Machado, "Systems of Navier-Stokes equations on Cantor sets," Mathematical Problems in Engineering, vol. 2013, Article ID 769724, 8 pages, 2013.

[23] X. J. Yang, Advanced Local Fractional Calculus and Its Applications, World Science, New York, NY, USA, 2012.

[24] X. J. Yang, H. M. Srivastava, J. H. He, and D. Baleanu, "Cantortype cylindrical-coordinate method for differential equations with local fractional derivatives," Physics Letters A, vol. 377, no. 28, pp. 1696-1700, 2013.

[25] M. Hu, D. Baleanu, and X. Yang, "One-phase problems for discontinuous heat transfer in fractal media," Mathematical Problems in Engineering, vol. 2013, Article ID 358473, 3 pages, 2013.

[26] A. M. Yang, C. Cattani, C. Zhang, G. N. Xie, and X. J. Yang, "Local fractional Fourier series solutions for non-homogeneous heat equations arising in fractal heat flow with local fractional derivative," Advances in Mechanical Engineering, vol. 2014, Article ID 514639, 5 pages, 2014.

[27] X. Yang and D. Baleanu, "Fractal heat conduction problem solved by local fractional variation iteration method," Thermal Science, vol. 17, no. 2, pp. 625-628, 2013.

[28] A. Yang, C. Cattani, H. Jafari, and X. Yang, "Analytical solutions of the one-dimensional heat equations arising in fractal transient conduction with local fractional derivative," Abstract and Applied Analysis, vol. 2013, Article ID 462535, 5 pages, 2013.

[29] C. F. Liu, S. S. Kong, and S. J. Yuan, "Reconstructive schemes for variational iteration method within Yang-Laplace transform with application to fractal heat conduction problem," Thermal Science, vol. 17, no. 3, pp. 715-721, 2013.

[30] A. M. Yang, J. Li, H. M. Srivastava, G. N. Xie, and X. J. Yang, "The local fractional Laplace variational iteration method for solving linear partial differential equations with local fractional derivatives," Discrete Dynamics in Nature and Society, vol. 2014, Article ID 365981, 2014.

[31] C. G. Zhao, A. M. Yang, H. Jafari, and A. Haghbin, "The YangLaplace transform for solving the IVPs with local fractional derivative," Abstract and Applied Analysis, vol. 2014, Article ID 386459, 5 pages, 2014.

[32] Y. Z. Zhang, A. M. Yang, and Y. Long, "Initial boundary value problem for fractal heat equation in the semi-infinite region by Yang-Laplace transform," Thermal Science, vol. 18, no. 2, pp. 677-681, 2014. 


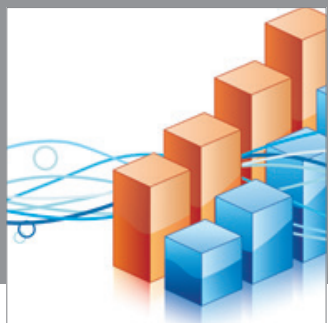

Advances in

Operations Research

mansans

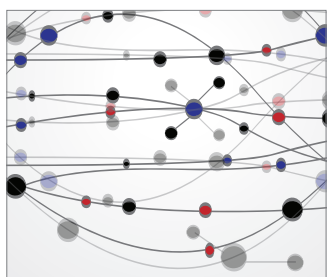

The Scientific World Journal
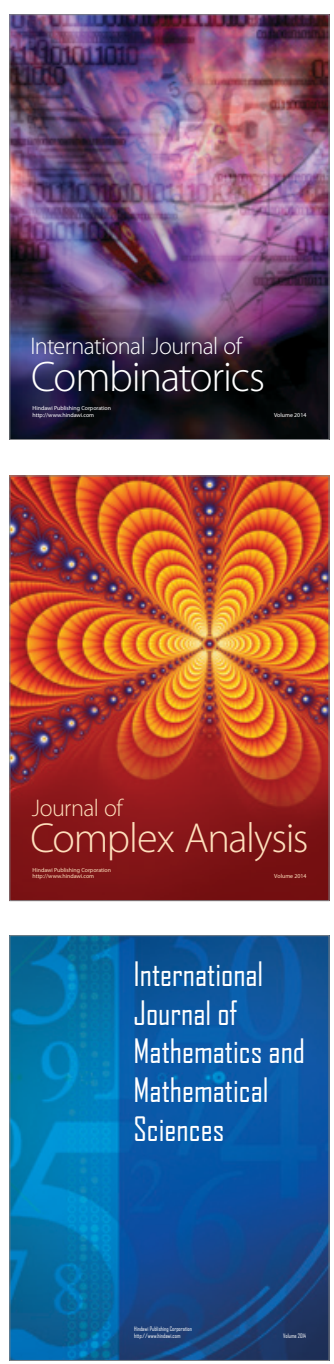
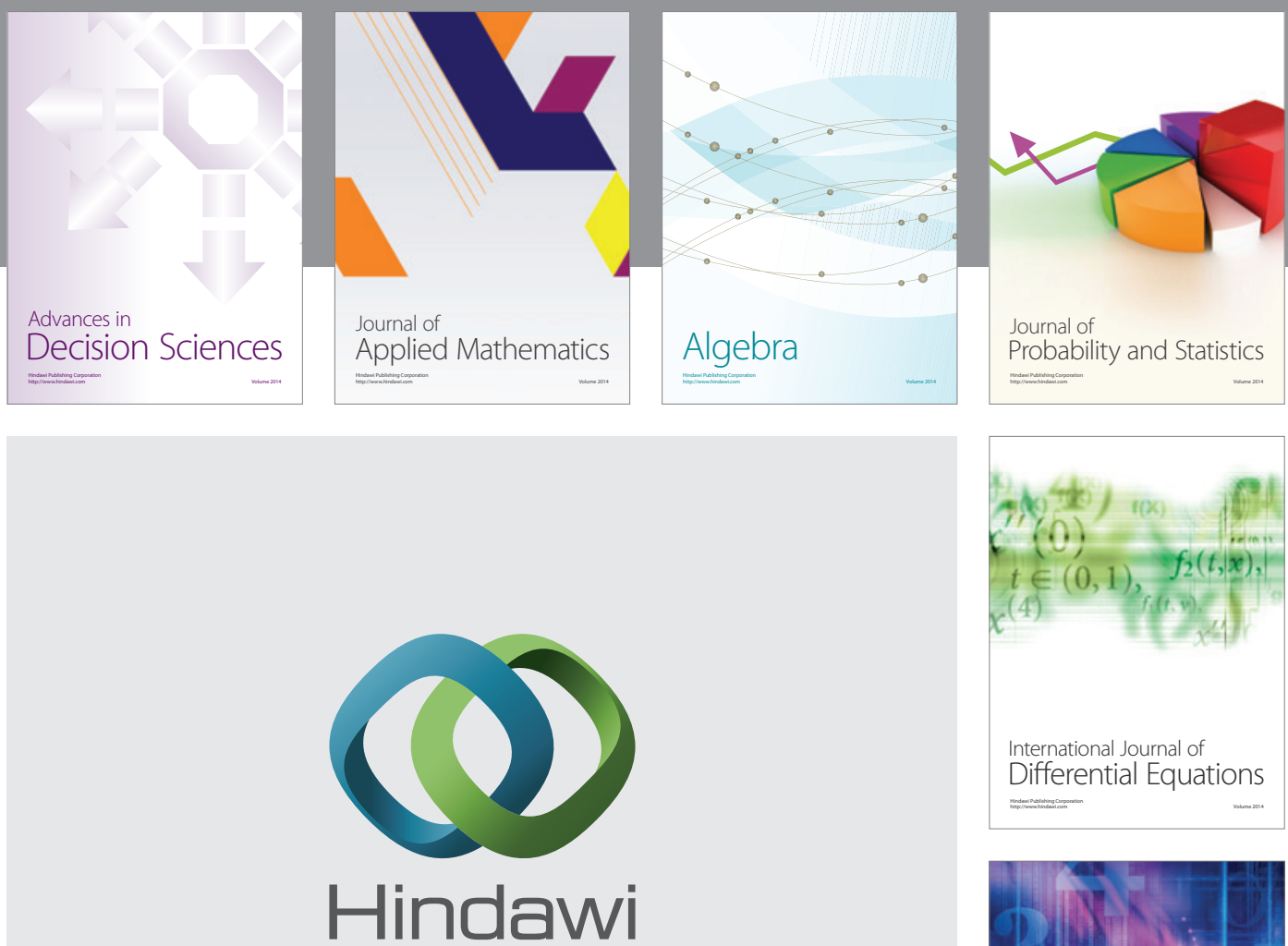

Submit your manuscripts at http://www.hindawi.com
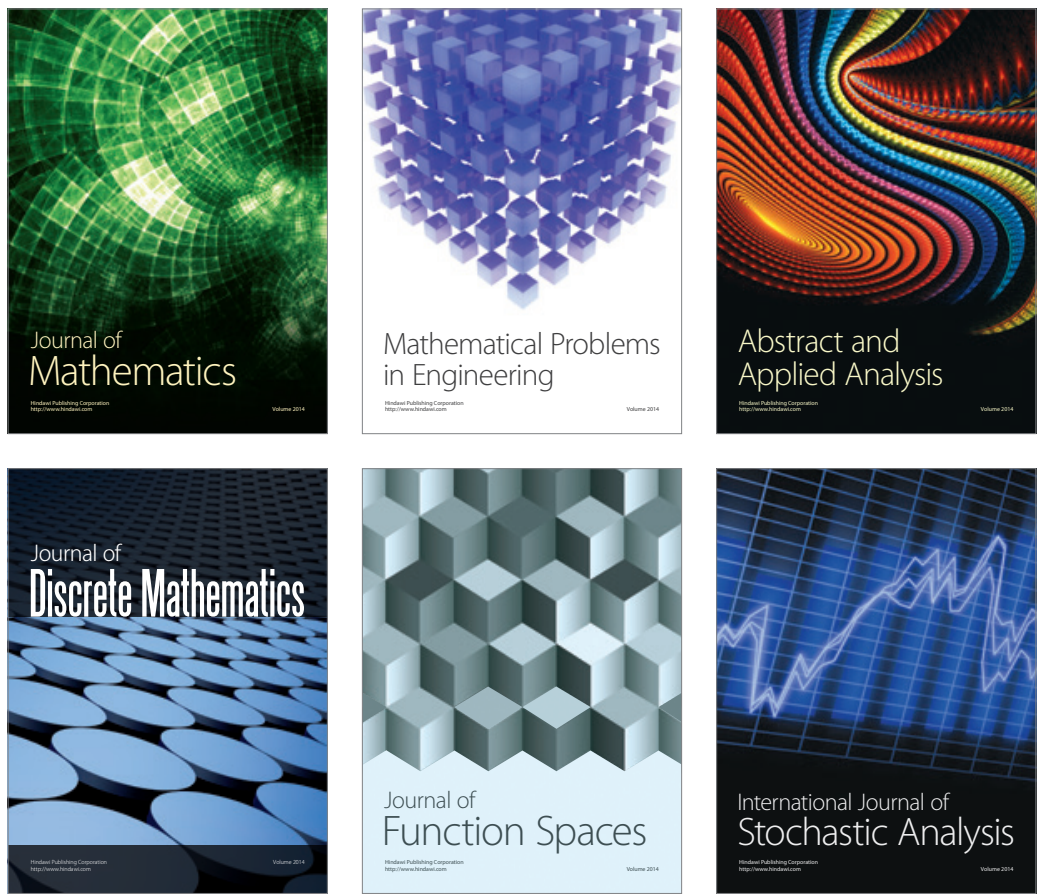

Journal of

Function Spaces

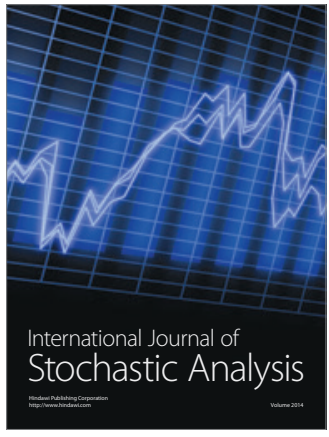

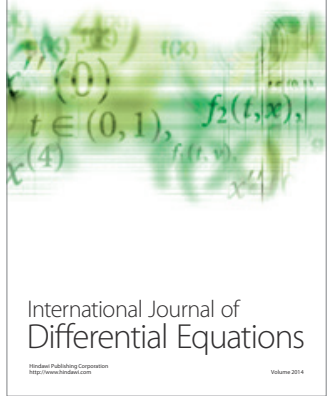
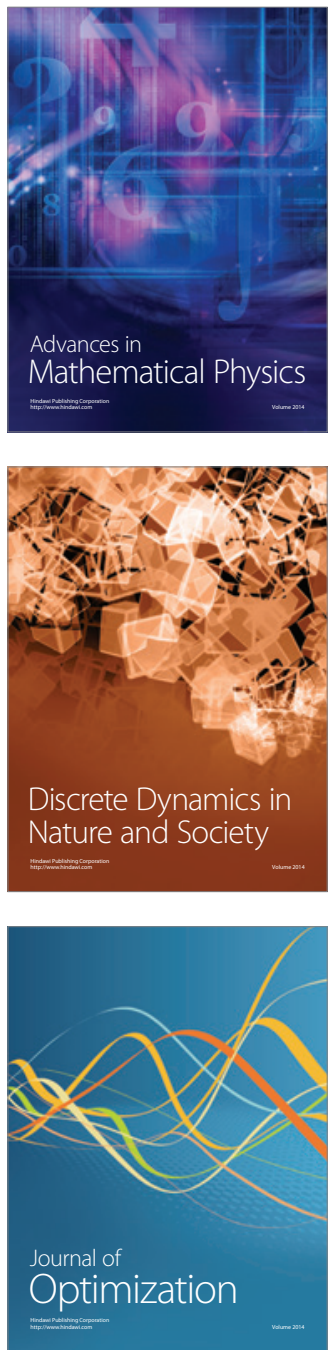